\title{
Aportes a la comprensión de la doble excepcionalidad: Alta capacidad con trastorno por déficit de atención y alta capacidad con trastorno del espectro autista
}

Conejeros-Solar, María Leonor 1; Gómez-Arizaga, María Paz 2; Sandoval-Rodríguez, Katia Geraldina 3; Cáceres-Serrano, Pablo Andrés 4

Aportes a la comprensión de la doble excepcionalidad: Alta capacidad con trastorno por déficit de atención y alta capacidad con trastorno del espectro autista

Revista Educación, vol. 42, núm. 2, 2018

Universidad de Costa Rica, Costa Rica

Disponible en: http://www.redalyc.org/articulo.oa?id=44055139016

DOI: https://doi.org/10.15517/revedu.v42i2.25430

Esta obra está bajo una Licencia Creative Commons Atribución-NoComercial-SinDerivar 3.0 Internacional. 


\title{
Aportes a la comprensión de la doble excepcionalidad: Alta capacidad con trastorno por déficit de atención y alta capacidad con trastorno del espectro autista
}

\author{
Contributions to the understanding of Twice Exceptionality: High Ability with Attention Deficit Disorder and \\ High Ability with Autism Spectrum Disorder
}

Maria Leonor 1 Conejeros-Solar [1]

Pontificia Universidad Católica de Valparaiso, Chile

DOI: https://doi.org/10.15517/revedu.v42i2.25430

leonor.conejeros@pucv.cl

Maria Paz 2 Gómez-Arizaga [2]

Universidad de Los Andes, Chile

mpgomez@uandes.cl

Katia Geraldina 3 Sandoval-Rodríguez [3]

Pontificia Universidad Católica de Valparaíso, Chile

katia.sandoval@pucv.cl

Pablo Andrés 4 Cáceres-Serrano [4]

Pontificia Universidad Católica de Valparaiso, Chile

pablo.caceres@pucv.cl

Recepción: 26 Junio 2016

Aprobación: 22 Abril 2018

\section{Resumen:}

En la década de los 80 surge un nuevo concepto en educación -doble excepcionalidad- que hace referencia a una paradoja entre una alta habilidad y una dificultad que impactan en el desarrollo y aprendizaje de niños, niñas y jóvenes. El objetivo del presente artículo es aportar a la comprensión de esta temática poco abordada en el contexto latinoamericano y a su estado de desarrollo para que profesionales que se vinculan al ámbito educativo la visibilicen e incorporen a sus prácticas pedagógicas. Se realizó una búsqueda sistemática en diversas bases de datos electrónicas y en revistas especializadas en el área de alta capacidad y se identificaron variables cognitivas y socioafectivas a la base de la condición de alta capacidad con trastorno por déficit de atención y alta capacidad con trastorno del espectro autista. Se incorporaron, además, aspectos relativos a identificación y apoyos educativos. Como principales temáticas se visualizan la complejidad en la comprensión de la doble condición, así como la necesidad de procesos de identificación e intervención comprensivos que reconozcan las características particulares de cada dualidad. Se recogen y comentan los principales

\section{NoTAS DE AUTOR}

[1] Psicóloga, Doctora en Educación. Profesora titular de la Escuela de Pedagogía, Pontificia Universidad Católica de Valparaíso. Entre sus líneas de investigación se encuentran la psicología educacional y cognitiva; y las altas capacidades, especialmente en lo referido a poblaciones vulnerables, estudiantado talentoso en educación superior, aspectos socioemocionales de niños, niñas y jóvenes talentos y doble excepcionalidad. Ha publicado artículos y capítulos de libro sobre altas capacidades. Participa como coeditora de la Revista Perspectiva Educacional. Formación de Profesores.

[2] Psicóloga, Pontificia Universidad Católica de Valparaíso, Ph.D. en Educación Especial con mención en dotación y talento, University of Arizona. Actualmente se desempeña como profesora investigadora en la Facultad de Educación de la Universidad de los Andes. Cuenta con amplia experiencia, a través de proyectos y publicaciones, en el área de talento académico, particularmente en las percepciones de estudiantes con alta capacidad acerca de sus experiencias y vivencias en contextos académicos.

[3] Educadora Diferencial, Magister en Educación mención Currículum, Pontificia Universidad Católica de Valparaíso (PUCV), Doctora en Ciencias de la Educación, Universidad de Granada. Docente de Escuela de Pedagogía de la PUCV, directora del Instituto de Desarrollo Cognitivo IDCO de Viña del Mar. Sus líneas de investigación son la identificación y caracterización personas doblemente excepcionales; o con trastornos de déficit de atención, aprendizaje y neurociencias.

[4] Psicólogo, Diploma de Estudios Avanzados y Máster en Metodología de las Ciencias del Comportamiento Universidad Complutense de Madrid. Actualmente es profesor de metodología de la investigación cuantitativa, metodología de la investigación cualitativa, teoría de la medición y análisis multivariante en la Pontificia Universidad Católica de Valparaíso. Realiza investigación el área de las altas capacidades cognitivas.

leonor.conejeros@pucv.cl 
desafíos tanto para su identificación como para su abordaje psicoeducativo y se plantean los aspectos que requieren de mayor investigación para avanzar en el estudio de esta temática. Es responsabilidad de los grupos profesionales de la educación visibilizar y atender a este estudiantado que convive con la incomprensión personal y social en entornos educativos que no los reconocen ni distinguen sus características.

Palabras ClaVe: Estudiante excepcional, atención, autismo, identificación, estrategias educativas.

\section{Abstract:}

In the 80 's a new concept emerges in education -Twice Exceptionality- that refers to a paradox between a high ability and difficulty that impacts the development and learning of children and young people.This article presents a review of the main the or etical contributions in this field. The goal of this article is to contribute to the understanding of this subject, which has not been addressed in the Latin American context, so that professionals linked to the educational field can consider and incorporate it to their pedagogical practices. A systematic search was conducted in several electronic databases and specialized journals in the field of giftedness, identifying cognitive and socio-affective variables based on the dual conditions of HighAbility with Attention Deficit Disorder and High Ability with Autistic Spectrum Disorder. In addition, aspects related to the identification and educational support were incorporated.The main research findings are related to the complexities for understanding both dual conditions, as well as the need for comprehensive identification and intervention processes that can recognize the unique characteristics of each condition. The main challenges are commented and discussed for identification and intervention purposes. The aspects that require further research are considered to make progress in the study of this subject. It is the responsibility of the education professionals to make visible and address the needs of this group of students who live with personal and social in comprehension in educational environments that do not recognize them and their characteristics.

KEYWORDs: Gifted students, Attention, Autism, Identification, Educational strategies.

\section{INTRODUCCIÓN}

Uno de los desafíos actuales a nivel pedagógico plantea la necesidad de considerar la diversidad del estudiantado, así como las barreras y facilitadores para el aprendizaje, lo que ha implicado concebir una educación para todas las personas, sin distinción (La Habana, UNESCO, 2002), incluso para quienes, por diversas razones, solo encuentran obstáculos y escasas oportunidades en los sistemas formales de educación, como es el caso de estudiantes que poseen una condición doblemente excepcional de aprendizaje, es decir, presentan simultáneamente una alta capacidad y una necesidad de apoyo (Gómez, Conejeros-Solar, Sandoval y Armijo, 2016).

Atender la doble excepcionalidad (en adelante 2e) requiere de aportes teóricos que permitan situar y poner en común la comprensión conceptual en la temática tanto a nivel de políticas públicas en educación como en salud en cada país.

En cuanto a la detección e identificación de estos niños y niñas, Kalbfleisch (2013) indica que el diagnóstico de la 2e no es ampliamente aceptado y apoyado en el contexto educativo convencional, pues existe poca información sobre su prevalencia y se extiende sobre un campo amplio de diversos tipos de dificultades. Sin embargo, los reportes empíricos y de investigación, disponibles en diversas revistas de corriente principal en el área, sustentan la necesidad de comprender y brindar atención a este grupo con capacidades y talentos únicos. En este sentido, Foley-Nicpon, Allmon, Sieck y Stinson (2011) realizaron una extensa revisión de las publicaciones en el área de la 2e en un período de 20 años (1991 a 2011). Concluyeron que la doble-excepcionalidad existe: La población de estudiantado dotado puede tener "una discapacidad coexistente" (p.13). Esta visión es reforzada por la National Association for Gifted Children en Estados Unidos (2009), al señalar que una discapacidad no excluye la presencia de una alta capacidad (en adelante $\mathrm{AC}$ ), con lo cual denota la complejidad que subyace a esta doble condición. Esta complejidad refuerza el objetivo de este artículo: Contribuir a la comprensión de esta temática y a su estado de desarrollo, para así poder constituir un aporte en este campo, en el que se considere que en Latinoamérica es aún un área incipiente que necesita ser abordada no solo a nivel académico, sino también por profesionales de las diferentes áreas de la educación. 


\section{Definición e historia del concepto}

Dos conceptos se encuentran a la base de la condición de 2e: el de AC y el de dificultad, dos extremos de habilidades y necesidades de apoyo que pueden generar conflictos académicos, sociales y emocionales (Baum, Schader y Owen, 2017; Trail, 2011). Su consideración, según Baum (2004), se vincula a una comprensión más amplia de la AC que se refleja en el informe Marland (1972) y sus modificaciones posteriores en el Congreso Norteamericano $(1978 ; 1988)$, que refieren no solamente a una AC cognitiva, sino también a una $\mathrm{AC}$ en áreas académicas específicas, como son las artes, liderazgo y creatividad. Definir la AC, dada la diversidad de modelos existentes en términos de su conceptualización, es una tarea compleja (Conejeros, Cáceres y Riveros, 2012). Para Pfeiffer (2015a) el grupo de estudiantes con AC presenta mayores probabilidades de conseguir logros extraordinarios en uno o más dominios culturalmente valorados en relación con otros grupos de estudiantes de la misma edad, experiencia y oportunidad, dado que aprenden a mayor velocidad, complejidad, profundidad y rapidez (Hébert, 2010; Pfeiffer, 2015b). Para Sternberg y Grigorenko (2002) los logros extraordinarios se deben a la combinación de habilidades analíticas, prácticas y creativas que este grupo utiliza para tener éxito en la vida.

Foley-Nicpon (2015) indica que la 2e en el contexto de la AC corresponde a una subárea de estudio. En este sentido, estudiantes con alguna discapacidad o necesidad de apoyo pueden, como cualquier otra persona, cumplir los criterios para que se le considere con AC, independiente de los desempeños deficientes que en algunas áreas pueda presentar (Lupart y Toy, 2009).

$\mathrm{Al}$ indagar en la historia de la 2e, los primeros indicios aparecen en los años 70, fruto del trabajo pionero de Elkind (1973) sobre un estudiante talentoso con dificultades de aprendizaje. Posteriormente, Withmore y Maker (1985) introducen la idea de que estudiantado con necesidades especiales también podía ser talentoso, lo que lleva a la consideración de la existencia de esta población y a su falta de atención educativa (Newman y Sternberg, 2004), en consecuencia, se avanza en la conceptualización de la "doble excepcionalidad" (2e) (Kalbfleish, 2013).

Reis, Baum y Burke (2014) proponen una nueva definición operacional de la 2e que incorpora los avances en investigación y la comprensión generada en la temática por una comisión compuesta por diverso personal académico, organizaciones y profesionales que se reunieron inicialmente en un simposio el año $2010 \mathrm{y}$ durante los últimos años con este fin.

La definición adoptada señala lo siguiente:

Los estudiantes doblemente excepcionales son estudiantes que demuestran un potencial de alto rendimiento o productividad
creativa en uno o más dominios tales como las matemáticas, la ciencia, la tecnología, las artes, lo visual, espacial, o las artes
escénicas o en otras áreas de la productividad humana y que manifiestan una o más discapacidades según la definición de los
criterios de elegibilidad estatales o federales. Estas discapacidades incluyen dificultades específicas de aprendizaje; trastornos
del habla y del lenguaje; trastornos emocionales/conductuales; discapacidades físicas; Trastornos del espectro autista; u otros
problemas de salud, tales como déficit de atención/hiperactividad. Estas discapacidades y altas capacidades se combinan
para producir una población única de estudiantes que pueden fallar, ya sea en demostrar un alto rendimiento académico
o discapacidades específicas. Sus dones pueden enmascarar sus discapacidades y sus discapacidades pueden enmascarar sus
dones. (Reis et al., 2014, p. 222)

A partir de esta definición, es posible señalar que estudiantes con 2e representan un grupo heterogéneo que incluye los distintos tipos de AC combinados con diversas necesidades de apoyo. Quizás por ello no hay consensos definidos en relación con su identificación (Trail, 2011). En este contexto, la identificación de la doble excepcionalidad resulta difícil de reconocer y conceptualizar, ya que representa una paradoja y trasciende las definiciones tradicionales de la AC. Asimismo, las implicaciones educativas son importantes, ya que el estudiantado que presenta 2e no cuenta con los apoyos adecuados para abordar sus necesidades educativas, ya que, entre otras cosas, al personal educador se le hace difícil comprender la presencia de una 
AC cuando este grupo de aprendices ha sido previamente diagnosticado con una necesidad de apoyo que se encuentra en el espectro de las dificultades (Foley-Nicpon et al., 2010a) .

\section{Manifestaciones de la doble excepcionalidad}

Frente a la necesidad de determinar certeramente el diagnóstico de la 2e para algunos de quienes han escrito sobre el tema, se hace necesario identificar la interacción entre las necesidades de apoyo y la AC, para lo cual se plantean 3 escenarios posibles. En primer lugar, uno en donde la dificultad enmascara a la AC, que tiene como consecuencia estudiantes con bajo o normal rendimiento académico. Aquí, pueden reconocerse por tener una necesidad de apoyo, pero no como quien posee una AC (Baum, Schader y Hébert, 2014). En segundo lugar, donde la AC enmascara la dificultad a través de estrategias compensatorias que permiten al estudiantado compensar sus dificultades con sus altas capacidades cognitivas, pues utiliza sus fortalezas de manera ventajosa, oculta sus dificultades y logra éxito en ambientes académicos desafiantes (Reis y Ruban, 2004). Un tercer escenario se produce cuando la AC y la dificultad interactúan recíprocamente, ocultándose entre sí. En este último escenario, el alumnado puede presentar un desempeño promedio en lo académico como en lo conductual, y resulta poco probable que sea reconocido por alguna de sus dos condiciones (Mullet y Rinn, 2015).

En cualquiera de estos tres casos, los procedimientos de detección recurren a múltiples instrumentos ya sea cognitivos, motivacionales y a otros sistemas como los autorreporte brindados por la población infantil y adolescente, informes de padres, profesorado y grupos de pares (Bélanger y Gagné, 2006; Heller, 2004; Manzano, Arranz y Sánchez de Miguel, 2010; Mullet y Rinn, 2015; Neal, Matson y Hattier, 2014; Peters y Engerrand, 2016).

Se comprende, entonces, que, en estas circunstancias, el diagnóstico que considere ambas condiciones conjuntamente llega a ser bastante complejo. En primer lugar, porque la definición de la condición doblemente excepcional aún está lejos de estar consolidada. Como señala Hughes (2011), el problema de la identificación de estos niños y niñas lleva a confusiones, pues AC y déficit dependen fuertemente de su definición, del contexto, del ambiente académico y de la percepción del profesorado. En segundo lugar, porque el estudiantado con doble excepcionalidad es extremadamente diverso y mientras una parte puede mostrar rasgos muy marcados, en otros casos estos atributos pueden pasar desapercibidos (Townend, Pendergast y Garvis, 2014). Además, tanto la AC como las dificultades son condiciones difíciles de reconocer, diagnosticar y detectar, tanto para profesionales especialistas y, en especial, para el profesorado de aula regular.

\section{Prevalencia de la doble excepcionalidad}

No se cuenta con datos de prevalencia en contextos de habla hispana, por lo que los datos que se presentan refieren a la realidad norteamericana. En este contexto, Foley-Nicpon, Assouline y Colangelo (2013) indican que es complejo brindar cifras en relación con la prevalencia, principalmente por la diversidad de modelos teóricos y prácticas de identificación, y porque en los contextos escolares tanto las habilidades como las dificultades pueden no estar formalmente reconocidas. Kalbfleisch (2013) plantea que además de saber muy poco sobre la prevalencia, en la población general existe escaso conocimiento de la incidencia, es decir, cuántas personas están actualmente diagnosticadas o identificadas como $2 \mathrm{e}$.

En este sentido, no existe un sistema que mida esta prevalencia en el sistema de salud o escolar (Foley Nicpon et al., 2010b). Otros autores como Ziemann (2009) señalan que se estima que hasta un 20\% del alumnado con AC podría presentar 2e, y en un estudio más reciente desarrollado por Barnard-Brak, Johnsen, Hannig y Wei (2015), se estima que el 9,1\% que presenta alguna discapacidad podrían también identificarse como estudiantes con alta capacidad. 
El objetivo de esta revisión es presentar los aportes empíricos actuales en el campo de la doble excepcionalidad, particularmente en lo referente a la manifestación dual de la alta capacidad con trastorno por déficit de atención (en adelante TDAH) y alta capacidad con trastorno del espectro autista (en adelante TEA). Se pretende, de esta forma, profundizar en las particulares características cognitivas y socioemocionales de ambas manifestaciones, identificando también nudos críticos y complejidades que subyacen a los procesos de identificación e intervención con este grupo en particular. Esto, con el fin de detectar posibles vacíos y direcciones futuras en investigación, así como también orientaciones para los grupos profesionales de la educación, en su vivencia diaria con este tipo de aprendices doblemente excepcionales.

Se escogen estos dos subtipos de doble excepcionalidad dado que el TDAH se presenta como el diagnóstico psiquiátrico más común en la infancia, con una prevalencia estimada, en Chile, de $10 \%$ en menores de 4 a 11 años con menos presencia en la adolescencia (De la Barra, Vicente, Saldivia y Melipillan, 2015). El TEA afecta, de manera severa, el desarrollo de los niños y las niñas (Neihart, 2000), y al presentar comportamientos tan inusuales, suele dificultar su diagnóstico, el cual con frecuencia resulta errado.. Además, ambos subtipos se caracterizan por dificultades en el funcionamiento ejecutivo, sobre todo para la resolución de situaciones problemáticas a nivel académico, social, personal, entre otros.

\section{Método}

Los artículos escogidos para esta revisión fueron seleccionados de múltiples maneras. En primer lugar, se utilizaron diferentes bases de datos online como Scopus, RedalyC, Scielo, Web of Science, Google Scholar y Science Direct. Esto, con el fin de obtener un cuerpo de artículos empíricos y revisados por pares en áreas de educación, educación especial, psicología educacional, salud, entre otros. En segundo lugar, para un foco temático más específico, se revisaron revistas especializadas en el área: Gifted Child Quarterly, Journal for the Education of the Gifted, Roeper Review, High Ability Studies, Gifted Education International, Journal of Advanced Academics y Revista de Altas Capacidades. En el proceso de búsqueda se incluyeron artículos tanto en inglés como en español, utilizando las siguientes palabras clave, ya sea de forma individual o de manera combinada: "doble excepcionalidad", "trastorno por déficit de atención", "trastorno del espectro autista", "autismo", "alta capacidad”, "altas habilidades", “sobredotación”. Tesis y artículos no revisados por pares fueron excluidos del estudio.

\section{Revisión empirica}

En los últimos 20 años, se encontraron 22 artículos empíricos que investigan la coocurrencia de AC-TDAH y AC-TEA, así como la identificación e intervenciones para estudiantes doblemente excepcionales.

\section{Doble excepcionalidad: Trastorno por déficit de atención con alta capacidad}

El diagnóstico de 2 e en el caso de estudiantes con AC y TDAH es complejo puesto que las manifestaciones del TDAH suelen ser similares a las de la AC, esto es: alto nivel de actividad, problemas en el control de impulsos, e impulsividad (Hartnett, Nelson y Rinn, 2004). El TDAH es considerado el trastorno del neurodesarrollo más frecuente, afecta diferentes escenarios de la vida de los niños, niñas y adolescentes (APA, 2013; Barkley, 2015). La American Psychological Association (2013) lo define como una pauta de comportamiento que persiste con características particulares de inatención o de hiperactividad-impulsividad y que interfiere en el desarrollo o en el funcionamiento. Como ya se planteó en el contexto chileno, la prevalencia del TDAH alcanza al 10\% entre menores de 4 a 11 años (Tapia, Veliz de Vos y Reyes, 2017; De la Barra, et al 2015). 
Una teoría que ha aportado a la comprensión de las manifestaciones de la AC -la cual originalmente no fue creada para este fin- es la de desintegración positiva de Dabrowski (1966), quien propuso que frente a diferentes estímulos internos y externos, pueden darse respuestas intensificadas a las que denominó sobreexcitabilidades. En el caso de estudiantes AC, investigaciones posteriores a Dabrowski encontraron que presentaban sobreexcitabilidades en el ámbito intelectual, imaginativo, y emocional (Daniels y Piechowski, 2009). Lee y Olenchak (2015), en una revisión exhaustiva de la bibliografía de AC y TDAH, afirman que dichas respuestas exacerbadas pueden traducirse en una intensificación de la actividad mental y la emocionalidad, distractibilidad y ensoñación, lo que es muy similar a los síntomas expresados por estudiantes con TDAH, y que puede, por tanto, llevar a confusión para la comprensión y diagnóstico de la dualidad AC-TDAH. Similar a estos resultados es lo encontrado por Rinn y Reynolds (2012), quienes hallaron una relación significativa entre los puntajes obtenidos por estudiantes con AC en sobreexcitabilidad imaginativa (ensoñación) y los indicadores clásicos de TDAH dados por el DSM IV y la Escala de Conners, lo cual puede traducirse en una tendencia en este grupo a manifestar sintomatología similar a TDAH, y llevar a errores en el diagnóstico.

Los estudios empíricos en torno a AC-TDAH han sido divididos en dos categorías: (a) características cognitivas y (b) características socioemocionales del estudiantado que presenta este subtipo de $2 \mathrm{e}$ (ver Tabla 1). 
TABLA 1

Estudios empíricos relacionados con estudiantes AC-TDAH

\begin{tabular}{llll}
\hline Referencia & Participantes & Método & Principales resultados \\
\hline $\begin{array}{l}\text { Antshel et al. } \\
(2007)\end{array}$ & $\begin{array}{l}\text { 92 estudiantes AC } \\
49 \text { estudiantes AC con TDAH }\end{array}$ & Cuantitativo & $\begin{array}{l}\text { Estudiantes AC-TDAH repetían más } \\
\text { cursos, tenian bajo desempeño en } \\
\text { bloques (WISC III) y presentaban } \\
\text { psicopatologia asociada. }\end{array}$ \\
\hline $\begin{array}{l}\text { Brown et al. } 117 \text { estudiantes AC-TDAH } \\
(2011)\end{array}$ & & Cuantitativo & $\begin{array}{l}\text { Bajo desempeño en 5 de } 8 \text { sub-escalas } \\
\text { de medición de funciones ejecutivas. }\end{array}$
\end{tabular}

\begin{tabular}{llll}
\hline $\begin{array}{l}\text { Foley Nicpon et } \\
\text { al. (2012) }\end{array}$ & 58 estudiantes AC & Cuantitativo & $\begin{array}{l}\text { Estudiantes AC-TDAH tenían } \\
\text { autoestima y autoconcepto }\end{array}$ \\
& & & $\begin{array}{l}\text { significativamente más bajos que } \\
\text { sus pares AC, y menor felicidad }\end{array}$ \\
& & & $\begin{array}{l}\text { sudiantes AC con TDAH } \\
\text { peribida. }\end{array}$
\end{tabular}

\begin{tabular}{|c|c|c|c|}
\hline $\begin{array}{l}\text { Fugate et al. } \\
(2013)\end{array}$ & $\begin{array}{l}20 \text { estudiantes } \mathrm{AC} \\
17 \text { estudiantes } \mathrm{AC} \text { con TDAH }\end{array}$ & Cuantitativo & $\begin{array}{l}\text { Estudiantes AC-TDAH con puntajes } \\
\text { significativamente más bajos que } \\
\text { pares AC en memoria de trabajo pero } \\
\text { mayor creatividad que estos. }\end{array}$ \\
\hline
\end{tabular}

\begin{tabular}{|c|c|c|c|}
\hline $\begin{array}{l}\text { Gómez et al. } \\
(2016)\end{array}$ & $\begin{array}{l}2 \text { estudiantes AC-TDAH } \\
2 \text { estudiantes AC-TEA }\end{array}$ & Cualitativo & $\begin{array}{l}\text { Estudiantes AC-TDAH presentan } \\
\text { visiones positivas de sí, se consideran } \\
\text { lideres, reconocen sus dificultades } \\
\text { frente a sus pares. }\end{array}$ \\
\hline
\end{tabular}

\begin{tabular}{|c|c|c|c|}
\hline $\begin{array}{l}\text { Moon et al. } \\
(2001)\end{array}$ & $\begin{array}{l}3 \text { estudiantes AC } \\
3 \text { estudiantes AC con TDAH } \\
3 \text { estudiantes TDAH } \\
\text { (solo varones) }\end{array}$ & Cualitativo & $\begin{array}{l}\text { Estudiantes AC-TDAH presentaban } \\
\text { dificultades en la regulación } \\
\text { emocional y relaciones con pares. } \\
\text { TDAH aparece como factor de riesgo } \\
\text { en el ámbito socioemocional. }\end{array}$ \\
\hline $\begin{array}{l}\text { Whitaker et al. } \\
(2015)\end{array}$ & $\begin{array}{l}39 \text { estudiantes AC } \\
30 \text { estudiantes AC con TDAH } \\
56 \text { estudiantes TDAH } \\
\text { (solo varones) }\end{array}$ & Cuantitativo & $\begin{array}{l}\text { Estudiantes AC-TDAH con puntajes } \\
\text { significativamente más bajos en } \\
\text { memoria verbal episódica que sus } \\
\text { pares AC y significativamente más } \\
\text { alta que estudiantes solo TDAH. }\end{array}$ \\
\hline $\begin{array}{l}\text { Zentall et al. } \\
(2001)\end{array}$ & $\begin{array}{l}3 \text { estudiantes AC } \\
3 \text { estudiantes AC con TDAH } \\
3 \text { estudiantes TDAH } \\
\text { (solo varones) }\end{array}$ & Cualitativo & $\begin{array}{l}\text { Estudiantes TDAH y AC-TDAH } \\
\text { presentaban dificultades en } \\
\text { atención, organización, escritura y } \\
\text { rendimiento escolar. } \\
\text { Estudiantes AC-TDAH mostraron } \\
\text { habilidades superiores en lectura y } \\
\text { creatividad. }\end{array}$ \\
\hline
\end{tabular}

\section{Caracteristicas cognitivas.}

Para una mejor comprensión de la manifestación de la AC con TDAH, es importante indicar que uno de los temas más complejos para entender esta comorbilidad es el rendimiento poco consistente que tienen estos estudiantes tanto en pruebas cognitivas como en el ámbito escolar, lo cual obstaculiza el poder apreciar sus reales habilidades. Antshel et al. (2007), con datos de un estudio longitudinal realizado en Estados Unidos, compararon el funcionamiento cognitivo y académico de estudiantes AC con y sin TDAH. Encontraron que estudiantes AC/TDAH repetían cursos más seguido, necesitaban de apoyos académicos a nivel escolar, y rendían más bajo en la subprueba Diseño con cubos del WISC III. De manera similar, Brown, Reichel y Quinlan (2011) midieron las funciones ejecutivas en una muestra de 117 estudiantes con AC que cumplían con los criterios diagnósticos para TDAH. Encontraron que la mayoría de estudiantes presentaban descensos 
en 5 o más de las medidas de funciones ejecutivas, así como puntajes bajos en memoria de trabajo, rapidez de procesamiento y memoria de trabajo verbal auditiva.

Zentall, Moon, Hall y Grskovic (2001) realizaron un estudio de casos múltiples de 9 estudiantes varones ( 3 solo TDAH, 3 solo AC y 3 AC-TDAH) para comparar sus habilidades académicas y de aprendizaje. Los resultados mostraron bajos niveles de atención para los grupos TDAH y AC-TDAH, así como también dificultades en el ámbito de la organización. Ambos grupos reportaron dificultades académicas frecuentes traducidas en un bajo rendimiento. Una problemática común era la escritura, no así la lectura, donde el grupo 2e demostró habilidades superiores. Por último, los estudiantes AC-TDAH se diferenciaban de sus pares TDAH en cuanto a la creatividad manifestada a través del humor, juegos, y juntar ideas de manera novedosa. Whitaker, Bell, Houtkamp y O'Callaghan (2015) midieron habilidades cognitivas y de memoria verbal en 125 participantes usando el mismo diseño que Zentall et al (2001): tres grupos comparativos, con predominancia de participantes hombres. El hallazgo más significativo dice que hay relación con la medición de la memoria verbal episódica, en donde los estudiantes AC-TDAH obtuvieron puntajes significativamente más bajos que sus pares AC y más altos que aquellos solo con TDAH, lo cual sugiere que las habilidades superiores podrían actuar como factor protector para las dificultades asociadas a TDAH.

Resultados semejantes encontraron Fugate, Zentall y Gentry (2013) en un estudio comparativo entre estudiantes AC con y sin TDAH. Los resultados indicaban un desempeño significativamente más bajo que sus pares sin TDAH en mediciones de memoria de trabajo, lo cual es consistente con la sintomatología típica de TDAH. Sin embargo, este mismo grupo de estudiantes puntuó más alto en creatividad, lo que, similar a los hallazgos de Zentall et al. (2001), da cuenta de que a pesar de las dificultades de retención de información, estudiantes AC-TDAH son capaces de un pensamiento altamente divergente que posibilita el pensamiento creativo.

\section{Características socioemocionales}

A partir de la revisión empírica, se puede apreciar que estudiantes solo diagnosticados con TDAH pueden manifestar baja autoestima, así como también un descenso en su autoconcepto cuando se evidencia una comorbilidad con una depresión (Barkley, 2015). Lo mismo se observa cuando existe una mayor severidad de los síntomas y un escaso apoyo a nivel escolar y social. Moon, Zentall, Grskovic, Hall y Stormont (2001), en un estudio con el mismo diseño muestral descrito en la en la sección anterior (i.e. 9 participantes con 3 condiciones) pero focalizado en el ámbito socioemocional, encontraron que estudiantes solo con AC tenían un buen ajuste emocional, adecuadas relaciones sociales, y fuertes vínculos a nivel familiar. Sin embargo, en el caso de la manifestación dual de AC con TDAH, los hallazgos apuntan a que estudiantes con 2e manifestaban mayores dificultades e intensidad emocional que sus pares solo con TDAH, incluso si han sido identificados en el ámbito de la AC. En este contexto, la identificación de la AC serviría para brindar servicios adecuados, pero no constituiría un factor protector, dado que este estudiantado tendría una mayor propensión a manifestar dificultades en el ámbito socioemocional.

Foley-Nicpon, Rickels, Assouline y Richards (2012b) encontraron diferencias significativas en la autoestima de estudiantes AC con diagnóstico dual de TDAH y sin este, donde el grupo AC-TDAH tenía el doble de posibilidades de reportar baja autoestima que estudiantes sin diagnóstico. Estos resultados apuntan a que la baja autoestima es una condición que está vinculada al diagnóstico de TDAH, lo cual podría influir en el desempeño académico actual o futuro de estudiantes con diagnóstico dual. Asimismo, las investigaciones encontraron importantes diferencias en el autoconcepto para ambos grupos. Estudiantes AC tenían una mejor percepción de su conducta y mayor felicidad percibida que aquellos grupos con AC y TDAH. Lo último surge como un resultado novedoso referente al grado en el cual el estudiantado juzga la calidad de su propia vida, lo que tendría un impacto negativo en este grupo particular de 2e. 
Gómez-Arizaga, Conejeros-Solar, Sandoval-Rodríguez y Armijo (2016), en un estudio cualitativo con estudiantes 2e, encontraron que, a nivel social y relacional, estudiantes AC y TDAH valoran la confianza y respeto a los vínculos que establecen tanto con sus pares como con sus docentes. Reconocen sus propias fortalezas para aprender y sus características individuales como liderazgo, curiosidad permanente y la necesidad de explorar lo desconocido. De igual forma, identifican las dificultades y barreras con que se encuentran, referidas al aburrimiento provocado por las experiencias educativas repetitivas y con baja novedad, y sus consecuencias en la consistencia y persistencia en el trabajo escolar

\section{Doble excepcionalidad: Trastorno del espectro autista con alta capacidad}

$\mathrm{Al}$ igual que la doble condición de $\mathrm{AC}$ con $\mathrm{TDAH}$, la comprensión del fenómeno de $\mathrm{AC}$ con TEA se torna compleja. A fines del siglo 20, se tenía ya alguna noción de que la AC y el TEA podrían compartir algunos rasgos y comportamientos (Cash, 1999). Se asumía también, que aquellas personas que se encontraban en la condición de autismo podrían evidenciar una serie de capacidades superiores (intelectuales, musicales, visuales entre otros).

El TEA refiere a un déficit persistente en torno a la comunicación e interacción social a través de diversos o múltiples contextos (APA, 2013; Foley-Nicpon, Fosenburg, Wurster y Assouline, 2017). Respecto a la prevalencia de personas con TEA, a nivel internacional, se menciona que, en los últimos años su frecuencia en Estados Unidos y otros países ha aumentado hasta cerca del 1\% de la población (APA, 2013). En el contexto chileno, la prevalencia de personas en situación de discapacidad por condición de autismo alcanza el $0.5 \%$ de la población (Ministerio de Desarrollo Social, 2015).

Actualmente, la prevalencia de la concomitancia de la AC con TEA sigue siendo incierta (Assouline, FoleyNicpon, Colangelo y O'Brien, 2008), pero se ha avanzado en la definición de los rasgos que caracterizan el perfil de esta (Burger-Veltmeijer, Minnaert y Van Den Bosch, 2015). Neihart (2000) señala que, al enfrentarse a un diagnóstico dual, es importante profundizar en las habilidades exhibidas por el estudiantado. Esto debido a que en el aspecto académico y cognitivo, estudiantes con AC pueden mostrar características comunes con el TEA, lo cual puede llevar a errores en la identificación. Estudiantes con AC, al igual que con TEA, pueden mostrar fascinación con las letras o los números. Sienten mucho interés por el aprendizaje a través de la repetición y pueden tender a obsesionarse con ciertos objetos y por temas que son de su interés (Little, 2002; Neihart, 2000). A pesar de estas semejanzas, la evidencia clínica da cuenta de que, a pesar de los puntos de encuentro, las dificultades a nivel sensorial, verbal y social en la dualidad AC-TEA son más severas y de tipo neurológico (Donnelly y Altman, 1994). Un aspecto que caracteriza al grupo que presenta la dualidad AC-TEA es que puede evidenciar un desempeño elevado e incluso brillante en sus estudios, mientras en otras áreas presentan un desempeño promedio (Neihart, 2000).

Resulta importante mencionar que existe poca evidencia de estudios controlados que den cuenta de las características de la dualidad AC-TEA, las que han sido recogidas mayoritariamente desde la experiencia clínica, teórica y anecdótica (Foley-Nicpon, Allmon, Sieck y Stinson, 2011). Al igual que en el caso de TDAH, se recoge la evidencia empírica en cuanto a características cognitivas y socioemocionales de esta dualidad, presentadas en la Tabla 2. 
TABLA 2

Estudios empíricos relacionados con estudiantes AC-TEA

\begin{tabular}{llll}
\hline Referencia & Participantes & Metodología & Resultados principales \\
\hline $\begin{array}{l}\text { Assouline et al. } \\
(2009)\end{array}$ & $\begin{array}{l}\text { 1 estudiante } \\
\text { AC-TEA } \\
1 \text { estudiante AC }\end{array}$ & Mixta & $\begin{array}{l}\text { Las principales diferencias entre } \\
\text { ambos grupos de estudiantes se } \\
\text { dieron en velocidad de procesamiento, } \\
\text { atención selectiva, distractibilidad e }\end{array}$ \\
& & $\begin{array}{l}\text { impulsividad, ámbitos en los cuales } \\
\text { la alumna con TEA obtuvo resultados } \\
\text { más bajos. También se encontraron } \\
\text { diferencias en el ámbito de la }\end{array}$ \\
& & $\begin{array}{l}\text { socialización. } \\
\end{array}$
\end{tabular}

Assouline et al. 59 estudiantes Cuantitativo (2012) AC-TEA

Foley-Nicpon et al. (2010a)

18 estudiantes
AC-TEA

Cuantitativo

Foley-Nicpon et al. (2012a)

52 estudiantes AC-TEA

Cuantitativo

Gómez et al. (2016)

$$
\begin{aligned}
& 2 \text { estudiantes } \\
& \text { AC-TDAH } \\
& 2 \text { estudiantes } \\
& \text { AC-TEA }
\end{aligned}
$$

$\begin{array}{lll}\begin{array}{l}\text { Melogno, et al. } \\ (2015)\end{array} & \begin{array}{l}1 \text { estudiante } \\ \text { AC-TEA }\end{array} & \text { Mixta } \\ \begin{array}{l}\text { Rubenstein et al. } \\ (2015)\end{array} & \begin{array}{l}30 \text { padres de } \\ \text { estudiantes } \\ \text { AC-TEA }\end{array} & \text { Cualitativo } \\ \end{array}$

Cualitativo

Se descubre una relación entre los bajos resultados en habilidades cognitivas básicas en estudiantes con AC-TEA como predictores de resultados en lectura, matemática y lenguaje escrito.

Se encontraron importantes discrepancias entre los resultados de habilidades cognitivas superiores $y$ básicas en estudiantes AC-TEA.

En estudiantes AC-TEA, se encuentran diferencias significativas entre los resultados de habilidades cognitivas superiores y básicas.

Estudiantes AC-TEA manifiestan tener pocos amigos, y buscan características como respeto y que los defiendan. Han sufrido bullying.

Estudiante AC-TEA obtiene bajos resultados en habilidades semánticas y altos resultados en habilidades gramaticales y fonológicas.

El estudio arroja que estudiantes con AC-TEA necesitan orden y estructura y que tienen dificultad para comunicarse, lo que afecta sus relaciones sociales.

\section{Caracteristicas cognitivas}

Algunos estudios que han explorado las características cognitivas de la dualidad AC-TEA se han focalizado en hacerlo de acuerdo con los puntajes obtenidos en la escala de Weschler. Foley-Nicpon, Assouline, Amend, y Schuler (2010b): describieron perfiles cognitivos de 18 estudiantes con AC/TEA. Encontraron que en habilidades verbales y no verbales, el grupo de estudiantes se situaban en el percentil 97, lo cual no ocurría 
con las habilidades básicas, en donde se situaban en percentiles promedio. Resultados similares encontraron Foley-Nicpon, Assouline y Stinson (2012a) en un estudio con 52 estudiantes con diagnóstico dual ACTEA con el fin de explorar sus perfiles académicos y cognitivos. Nuevamente, se evidencian diferencias entre los puntajes obtenidos en habilidades cognitivas superiores (verbales y no verbales) y básicas (en particular memoria de trabajo y velocidad de procesamiento), lo que sugiere que, si bien esto es común en estudiantes AC el tamaño de la discrepancia, este es mayor a lo esperado y común a otros tipos de 2e. Un estudio de casos realizado por Assouline, Foley Nicpon y Doobay (2009), el cual comparó los perfiles de dos niñas AC una con y otra sin TEA, encontró resultados similares, en donde la participante con diagnóstico dual AC-TEA obtuvo resultados más bajos en velocidad de procesamiento. También se encontraron diferencias importantes en las funciones ejecutivas, donde la estudiante AC-TEA demostró importantes dificultades en atención selectiva, distractibilidad e impulsividad. Consistentes con lo encontrado por los estudios anteriores fueron las discrepancias en el instrumento de Weschler descritas por Melogno, Pinto y Levi (2015) en su estudio de caso de un niño AC-TEA.

En cuanto a rendimiento en áreas académicas tales como lectura, matemática, lenguaje escrito y oral, la investigación de Foley-Nicpon et al. (2010) da cuenta de que estudiantes AC-TEA tenían un rendimiento muy superior en comparación a sus pares de la misma edad en velocidad y comprensión de lectura. En escritura, si bien igualaban a sus pares en cuanto a la creación rápida de oraciones, en deletreo y escritura creativa eran muy superiores. Asimismo, en esta área evidenciaron dificultades en su motricidad fina. En matemática, demostraron habilidades superiores en cálculo y resolución de problemas, no así en responder rápidamente a operaciones matemáticas simples. En el estudio de Assouline et al. (2009), los resultados en rendimiento de las dos participantes fue muy alto, con una sola diferencia significativa en la estudiante AC-TEA, referida a recordar historias, una sub-prueba del lenguaje oral. En el estudio de caso realizado por Melogno, Pinto y Levi (2015), se midieron las habilidades metalingüísticas del participante, es decir, reflexiones en torno a la estructura del lenguaje (ej.morfología, sintaxis). Se obtuvieron resultados muy poco homogéneos en esta medición, que sitúan al participante en los extremos del desempeño (muy alto-muy bajo). Los puntajes más bajos fueron en el nivel metalingüístico explícito en donde se debe trabajar con sistemas "abiertos" de mayor ambigüedad e incertidumbre, dando cuenta de la tendencia de rasgos TEA de preferir sistemas cerrados con más lógica y estructura.

Por último, una investigación realizada por Assouline, Foley Nicpon y Dockery (2012) con 59 estudiantes con la doble condición AC-TEA, encontró una asociación positiva entre las características cognitivas y el rendimiento académico de este grupo de estudiantes. Particularmente, los bajos resultados en habilidades cognitivas básicas predecían los resultados en lectura, matemática y lenguaje escrito.

\section{Caracteristicas socioemocionales}

En cuanto al ámbito socioemocional, los estudios en AC-TEA dan cuenta de las dificultades que emergen en este grupo y evidencian las características típicamente asociadas a TEA. Un ejemplo de este tipo de hallazgos es el estudio de Foley Nicpon et al. (2010a) donde, además de medidas cognitivas, se analizaron los perfiles socioemocionales de estudiantes AC-TEA, a través de medidas de conducta adaptativa y mediante observación de profesionales y reportes de padres, madres o personal cuidador. Se encontró que, si bien este estudiantado reportaba resultados promedio en comunicación y vida cotidiana, en socialización tenía puntajes bajos, discrepancia que es típicamente observada en casos TEA. Asimismo, las observaciones y reportes dieron cuenta de dificultades en la interacción social recíproca, particularmente en expresiones faciales, responder a conversaciones e involucrarse en juego de tipo imaginativo con pares. Asimismo, se encontraron dificultades en los cambios de rutina y en compartir lo propio con otros. No obstante, no se encontró la presencia de conductas estereotipadas, preocupaciones atípicas, rituales verbales, entre otros 
rasgos que se pueden encontrar en TEA. En el ámbito escolar, el estudiantado no reportó grandes dificultades, ni estrés social, se encontró, incluso, un nivel de autoestima adecuada.

El estudio de casos comparativo de Assouline et al. (2009) con dos estudiantes AC (con y sin diagnóstico de TEA) también reportó dificultades en el ámbito socioemocional, en donde la estudiante AC-TEA mostró algunos problemas relacionados con: la expresión emocional, el juego creativo e imaginativo, el uso de comunicación no verbal y gestos, la comunicación recíproca, así como también dificultad para involucrarse con pares de su misma edad y escasa empatía. También se reportaron altos niveles de dificultades psicosociales (ej. depresión). Por otro lado, la misma estudiante presentaba adecuados niveles de autocontrol y cooperación con otros y, similar a la investigación anterior, no se encontraron tendencias hacia conductas estereotipadas e intereses restringidos.

Una investigación cualitativa realizada por Rubenstein, Schelling, Wilczynski y Hooks (2015), en donde los padres y madres reportaron sobre las conductas de sus hijos e hijas, arrojó resultados similares a los descritos anteriormente. Encontraron que una de las mayores dificultades en el grupo AC-TEA eran las habilidades sociales, particularmente la escasa capacidad de comunicarse de manera convencional, lo cual, a su vez, afectaba su entorno, particularmente la relación con sus docentes. Otro rasgo relevante era la necesidad de orden y estructura, no solo en el ámbito académico, sino que en todas las áreas de la vida cotidiana, en donde la predictibilidad de los hechos futuros era crítica para un buen funcionamiento socioemocional.

Por último, en la misma línea de estudios cualitativos se encuentra el realizado por Gómez et al. (2016), donde se exploraron, de manera cualitativa, las relaciones de estudiantes con su contexto escolar inmediato. En el caso de estudiantes AC-TEA, se encontró que tenían pocos amigos, pero que en ellos buscaban una relación de respeto y también de protección, debido a situaciones de bullying vividas en el pasado. Asimismo, eran conscientes de su dificultad (no necesariamente de su AC) y algunas veces se consideraban líderes de su grupo de pares.

\section{Identificación y apoyos educativos para la doble excepcionalidad}

Se encontraron estudios focalizados en la identificación de estudiantes 2e, particularmente en los desafíos y complejidades que presenta este proceso. Asimismo, se presentan investigaciones empíricas en cuanto a intervenciones efectivas en el ámbito de la doble excepcionalidad. El resumen de estas investigaciones se puede encontrar en la Tabla 3.

\section{Identificación}

Tal vez uno de los procesos más complejos y dificultosos en relación con la temática de la doble excepcionalidad es la identificación de este grupo de estudiantes. Por un lado, están las construcciones sociales en torno a esta paradoja, las cuales conllevan a la invisibilización del fenómeno, dado que la persona es reconocida (y muchas veces atendida) en sus dificultades, pero no necesariamente por sus capacidades, aspecto usualmente ignorado por el profesorado e incluso por los padres y madres (Kalbfleisch 2013; Silverman, 1998).

Por otra parte se encuentra la complejidad de evaluar de manera adecuada a estudiantes que presentan 2e, debido a la tendencia en educación y prácticas clínicas de encasillar los resultados -tanto de la AC como de las dificultades- de manera prototípica, sin considerar que la expresión de la $\mathrm{AC}$ puede ser muy diferente a la norma entregada por los instrumentos de evaluación, la cual se sitúa generalmente en niveles promedio e incluso bajo en evaluaciones estandarizadas (Kalbfleisch y Iguchi, 2008). Algunos estudios plantean, por ejemplo, que el desempeño de estudiantes con 2e en la Escala de Inteligencia de Weschler para Niños (WISC) no es siempre igual al de sus pares con AC sin discapacidad asociada, y que tomar como puntaje de corte 
el coeficiente intelectual dado por la escala total de este instrumento es contraproducente, debido a las discrepancias que manifiesta este grupo de estudiantes en cuanto a sus desempeños en las distintas subpruebas (Krochak y Ryan, 2007). Por esto los equipos expertos en 2e recomiendan no equiparar al estudiantado respecto a una norma estandarizada, sino que más bien la comparación debe ser intrapersonal, considerando sus fortalezas y debilidades (Silverman, 1998).

McCallum, Bell, Coles, Miller, Hopkins y Hilton-Prillhart (2013) encontraron que el foco exclusivo en el rendimiento como criterio de identificación es erróneo, debido a la disparidad en el rendimiento académico de estudiantes con 2e, el cual no alcanza lo esperable para un estudiante con AC; por el contrario, puede llegar a ser muy fluctuante y variable a través del tiempo.

Otra problemática que emerge desde la identificación con orientación tradicional o estandarizada, encuentra relación con los protocolos de administración de los instrumentos, que muchas veces son rígidos en cuanto a factores como tiempo o reformulación de ítems de carácter oral, con lo cual también se puede ver afectado el desempeño de los estudiantes con 2e, dependiendo de la dificultad asociada. Conejeros-Solar, Gómez-Arízaga, Sandoval-Rodríguez, Cáceres, Armijo y Abello (2016) encontraron que los protocolos de administración del Test de Matrices Progresivas de Raven debían modificarse. Por ejemplo, en el caso de estudiantes con TEA, se realizaron adaptaciones como respetar los tiempos de respuesta de estudiantes y en el caso de TDAH, repetir instrucciones cuando fuese necesario y monitorear al estudiantado durante la administración del test para asegurarse que permaneciera centrado en la tarea.

En este contexto, nacen, desde la bibliografía anglosajona, los conceptos de baja identificación (underidentification) e identificación errónea (misidentification) que se refieren, por un lado, a la escasa posibilidad de los estudiantes con 2e de ser nominados para programas de atención a la AC, y por otro, al fenómeno relacionado con la rotulación errónea que se realiza a partir de inferencias de observaciones de las conductas manifestadas por el estudiantado, pero que no consideran el contexto ni la evidencia empírica en torno a la doble excepcionalidad (Reis et al., 2014).

\section{Intervenciones}

En cuanto al apoyo que se otorga a nivel educativo o clínico en la temática de la 2e, antes descrita, diversas investigaciones refieren que es muy difícil que el profesorado y otros actores educativos consideren a estudiantes con $2 \mathrm{e}$ para que estos grupos sean evaluados y puedan participar en programas especiales de enriquecimiento para la AC (Bianco y Leech, 2010; Foley-Nicpon et al., 2010; Reis et al., 2014; Ruban y Reis, 2005). Barnard-Brak, Johnsen, Pond Hannig y Wei (2015), en su estudio longitudinal de educación especial, refieren que 300 estudiantes que presentaban 2e de una muestra de 13.176 niños con algún diagnóstico en particular, solo el $11.1 \%$ asistía a programas y educación para niñez con talento, puesto que no se reconocían por su alta capacidad sino que esta se encontraba enmascarada. Habitualmente, lo que se entrega es un servicio "parcelado", es decir, el estudiantado solo es atendido en sus dificultades, quedando la AC relegada e invisibilizada. Sin embargo, se ha visto que también puede ocurrir lo contrario, aunque de manera menos frecuente. Debido a la ocurrencia de la compensación de habilidades que enmascaran las dificultades, puede ocurrir que el estudiantado sea reconocido solamente por su AC y se ignore -durante mucho tiempo- la presencia de una necesidad de apoyo sin que se le otorgue la atención necesaria (McCallum et al., 2013).

Baum, Schader y Hébert (2014) exploraron, de forma cualitativa, las experiencias de 10 estudiantes con 2e quienes ingresaron a la educación secundaria y formaban parte de un programa basado en las fortalezas, asentado en un establecimiento educacional. Dicho modelo tenía como característica principal, intervenciones realizadas con el estudiantado tanto a nivel académico (diferenciación, foco en habilidades) como socioemocional, con especial énfasis en el bienestar psicosocial. Estudiantes participantes reportaron ganancias en lo académico (ej. mayor motivación por terminar proyectos) y crecimiento en aspectos socioemocionales, pasando de estados de gran ansiedad y desesperanza aprendida, a sentirse con mayor 
confianza hacia el término de su escolaridad y comienzo de la educación terciaria. Asimismo, se obtuvieron como resultados de este estudio pautas para la creación de programas adecuados en donde el estudiantado se sienta seguro, cómodo, desafiado, valorado y aceptado en su doble condición.

Shultz (2012), por su parte, investigó las valoraciones y apreciaciones de 36 participantes, entre quienes estaban padres, madres, docentes y personal de consejería escolar de estudiantes 2e que asistían a la educación secundaria de cinco escuela públicas de Nueva York. Se exploró el impacto, en términos de barreras y oportunidades, de cursos de Colocación Avanzada (Advanced Placement) y cursos para obtener créditos universitarios. Los hallazgos apuntan a que, si bien se trata de una instancia escolar beneficiosa para estudiantes 2e, ya que les permite acceder a otro campo del conocimiento y generar oportunidades que permitan su acceso futuro a la universidad, también se pueden encontrar dificultades, entre las cuales se anotan las escasas adecuaciones realizadas por el profesorado de este tipo de cursos. A pesar de los obstáculos, el estudiantado valoraba la presencia de personal mentor en su etapa escolar que actuaba como guía para el futuro y mostraba respeto por sus habilidades.

Koshy y Pascal (2011) reportaron los resultados de una investigación acción (I-A) con escuelas y docentes de estudiantes en rangos etarios de 4 a 7 años, quienes implementaron adecuaciones para estudiantes con $\mathrm{AC}$ en el aula escolar. El profesorado participante reportó la creación de planes en conjunto en diversos aspectos: identificación de estudiantes con AC, implementación de proyectos de enriquecimiento basados en el desarrollo de habilidades superiores (ej. creatividad y resolución de problemas), revisión y adaptación de las metodologías de enseñanza focalizándose en los intereses y pasiones de sus estudiantes, mejoras en las prácticas evaluativas y mayor integración de los padres y madres en el proceso educativo. Asimismo, este extenso proyecto de I-A reportó en algunos establecimientos un especial foco en $2 \mathrm{e}$, en donde se diseñaron actividades especiales para que estudiantes $2 \mathrm{e}$ pudiesen exhibir sus fortalezas a través de actividades "handson", lo cual ayudó también a poder identificarles cuando su AC estaba enmascarada por las dificultades.

Los estudios de corte empírico que hacen alusión a la intervención educativa o psicoeducativa de las personas con la condición de 2e son muy escasos, la mayoría de la investigación en esta temática alude a la identificación y caracterización de la 2e, sin embargo, a continuación se muestran algunos (ver Tabla 3 ) 
TABLA 3

Estudios empíricos relacionados con la atención o intervención educativa a la doble excepcionalidad

\begin{tabular}{llll}
\hline Referencia & Participantes & Metodologia & Resultados principales \\
\hline $\begin{array}{l}\text { Barnard-Brak et al. } \\
\text { 2015) }\end{array}$ & 1.376 & $\begin{array}{l}\text { Estudio } \\
\text { Longitudinal }\end{array}$ & $\begin{array}{l}\text { Los principales hallazgos se orientan } \\
\text { a la falta de oportunidades de } \\
\text { intervención y desarrollo del talento } \\
\text { en el estudiantado con } 2 \mathrm{e} \text {, por la } \\
\text { invisibilización que esta reviste. Se } \\
\text { sugiere además que es necesaria } \\
\text { la consideración de estrategias de } \\
\text { identificación y atención a través de } \\
\text { políticas públicas }\end{array}$ \\
& &
\end{tabular}

Se identifican cinco factores esenciales al desarrollo de estudiante 2e: seguridad psicológica, tolerancia a la asincronia, tiempo, relaciones positivas $y$ el uso consistente de un enfoque basado en fortalezas y talentos.

Bianco y Leech. (2010)

Foley-Nicpon et al., (2010a)

MeCallum et al. 2013

1.242 estudiantes de tercer grado
277 docentes $(52$
educación especial

195 de educación

general y 30 educación para la alta capacidad)

18 estudiantes AC-TEA
Estudio Mixto

Similitudes entre todo el personal docente a una predisposición menor para promocionar a estudiantes $2 \mathrm{e}$ a asistir a programas de alta capacidad, por el hecho de presentar un diagnóstico de alguna discapacidad en forma conjunta.

Cuantitativo

Las intervenciones terapéuticas que parecen más prometedoras para estudiantes con TEA incluyen intervenciones cognitivo-conductuales, a pesar de que la revisión de su eficacia en estudiantado dotado con TEA es inexistente. Las intervenciones psicoeducativas efectivas adoptan un enfoque multinivel, donde se programa con base en las fortalezas cognitivas y académicas, al mismo tiempo que se considera la constelación de dificultades de la persona a nivel comunicacional, social, y conductual.

Cuantitativo

Se realiza un screening para reconocer estudiantado $2 \mathrm{e}$ desde el paradigma de respuesta a la intervención en el contexto del curriculo.
Shultz (2012)

Koshy y Pascal (2011)

\begin{tabular}{|c|c|}
\hline $\begin{array}{l}36 \text { participantes } \\
\text { (12 padres y } \\
\text { madres } 12 \\
\text { docentes, } 6 \\
\text { personal de } \\
\text { consejeria escolar } \\
\text { y } 6 \text { estudiantes } 2 \text { e) }\end{array}$ & $\begin{array}{l}\text { Cualitativo } \\
\text { Entrevistas }\end{array}$ \\
\hline $\begin{array}{l}6 \text { participantes } \\
42 \text { docentes y } 14 \\
\text { gersonal consejero) }\end{array}$ & $\begin{array}{l}\text { Investigación } \\
\text { Acción }\end{array}$ \\
\hline
\end{tabular}

Los resultados de este estudio indicaron que la cultura escolar y las decisiones tempranas de inserción afectan la inscripción de estudiantado secundario a programas de colocación avanzada en las universidades.

Se constató en qué situación se encontraban los diferentes programas de educación de talento. Los hallazgos del proyecto demostraron la necesidad de atender la diversidad intereses y motivaciones del estudiantado $2 \mathrm{e}$ de forma que se promuevan oportunidades para su desarrollo desde muy pequeño en las escuelas. 


\section{CONCLUSIONES}

Probablemente, una de las mayores dificultades en el ámbito de la AC está vinculada con dejar atrás las concepciones más tradicionalistas y abrir el espectro a distintas manifestaciones de esta, como es el caso de la 2e. A lo largo de la historia de la AC, en general, en lo que se refiere a las representaciones sociales en cuanto al constructo (Tavani, Zenasni y Pereira-Fradin, 2009), ha existido una predominancia de conceptualizaciones "clásicas" y una tendencia a visiones homogeneizantes y estereotípicas en cuanto a las características de este grupo de estudiantes, las cuales priorizan rasgos como el coeficiente intelectual (CI), el alto rendimiento, entre otras.

La evidencia presentada en esta revisión, en cambio, apunta a la coexistencia real de dos condiciones contrapuestas (Foley Nicpon et al., 2011) que viene a ampliar las visiones más tradicionalistas de la alta capacidad mediante la incorporación del concepto de doble excepcionalidad. Sin embargo, adoptar esta nueva conceptualización implica también complejizar los ámbitos de la caracterización, identificación e intervenciones apropiadas para este grupo de estudiantes. En este sentido, si bien se encontró un cuerpo razonable de investigaciones en el ámbito de las 2e y en los dos subtipos abordados, aún existen vacíos (Lee y Olenchak, 2015) y se puede apreciar una considerable variabilidad en los estudios existentes en cuanto a instrumentos y métodos utilizados. Asimismo, muchas de las investigaciones siguen usando el cociente intelectual (CI) en la identificación de la AC, y este no es el instrumento más sensible a las características de la doble excepcionalidad.

Sin embargo, se aprecian elementos comunes o patrones en cuanto a la caracterización de habilidades cognitivas y socioemocionales en estudiantes AC con TDAH y AC con TEA. Desde lo cognitivo y escolar, lo más relevante resulta la disparidad en las habilidades, así como desempeños académicos erráticos que no siguen un patrón conocido, ya sea para la alta capacidad o la necesidad de apoyo, y que, por lo tanto, causan desconcierto tanto en profesionales de la salud, educación, e incluso a los propias familias. Destacan en este ámbito lo referente al descenso en la memoria de trabajo y los altos niveles de creatividad en el caso de AC-TDAH, los altos puntajes en habilidades cognitivas superiores de los estudiantes AC-TEA versus desempeños descendidos en habilidades básicas.

En lo referente al ámbito socioemocional, surge como distintivo, para la dualidad AC-TDAH, la presencia de problemáticas en el plano psicológico, como una mayor posibilidad de presentar una baja autoestima y menor satisfacción con la vida derivada de la condición dual. Por otro lado, en el caso AC-TEA, resulta relevante considerar que, si bien hay presencia de rasgos típicamente asociados a TEA tales como dificultades en la socialización y en la interacción social, no aparecen, por ejemplo, conductas estereotipadas e incluso se observan altos niveles de adecuación y ajuste en el medio escolar, incluida la presencia de pequeños grupos de pares.

Implicancias para la investigación. Es importante la realización de mayor investigación empírica en la temática, a fin de indagar en las necesidades, barreras y facilitadores al aprendizaje y desarrollo de esta población. A pesar de numerosas investigaciones, principalmente en el ámbito anglosajón, aún existen vacíos, por ejemplo, en lo referente a la manifestación dual, que en el caso de este artículo se focalizó en la alta capacidad con TDAH o TEA. Asimismo, el espectro de manifestaciones de la 2e puede llegar a ser muy diverso, por lo que en futuras investigaciones también es importante incorporar otras dificultades concomitantes a la $\mathrm{AC}$, que pueden encontrarse en los ámbitos emocionales, relacionales y de aprendizaje.

A nivel contextual, si bien hay estudios con diversas mediciones en el ámbito familiar y escolar, resulta importante recoger, desde perspectivas cualitativas, las voces del propio estudiantado y sus familias, ya que si bien los cuestionarios de autorreporte utilizados en diversas investigaciones permiten trabajar con muestras más amplias, los enfoques interpretativos permiten contar con una visión más auténtica de sus experiencias, bienestar subjetivo, necesidades, proyecciones, entre otras. En este ejercicio, es también necesario recoger las 
perspectivas de otros agentes como el profesorado, los compañeros y compañeras de curso y profesionales de apoyo. Asimismo, la realización de estudios longitudinales que permitan realizar seguimientos al desarrollo de este grupo de estudiantes, podrían aportar luces sobre los apoyos a brindar y la estimulación de su potencial.

En el caso de la realidad latinoamericana, es indispensable iniciar estudios acerca de la situación de los estudiantes con 2e, considerando también a quienes se encuentran en condiciones de vulnerabilidad psicosocial y para quienes existen menores oportunidades de reconocimiento en su doble condición. En el caso de Chile, se hace necesario también explorar la temática, ya que el excesivo foco en los déficits (MINEDUC, 2009) centra los apoyos en la compensación de las dificultades y no en la promoción de las capacidades.

Implicancias para la práctica educativa. La condición de 2e impacta el desarrollo de niños, niñas y jóvenes; sin embargo, el desconocimiento de esta por parte de las políticas públicas, impiden su reconocimiento, su atención y su desarrollo. En la mayoría de los países hispanohablantes y en el caso de Chile, en particular, esta condición es invisible. La visibilización implica, entonces, incorporar esta temática en la formación inicial del profesorado, y profesionales de la psicología, psicología educativa y de las áreas de la salud y que trabajan con población infantil y adolescente.

Desde el ámbito de la identificación, se hace necesario contar con evaluaciones comprensivas y profundas que permitan caracterizar las distintas expresiones y los rasgos distintivos de la 2e, considerando un abanico amplio y uso de una multiplicidad de fuentes de información, desde una mirada holística más que reduccionista del fenómeno. Asimismo, es necesario flexibilizar la interpretación de resultados sobre todo cuando se usan instrumentos con normas, se debe abogar por interpretaciones más cualitativas centradas en el perfil del estudiante 2e, pues este es, probablemente, el caso más emblemático del uso de las escalas de Weschler, cuya interpretación "tradicional" no es posible para conocer las capacidades reales de los estudiantes 2e. Dadas las dificultades presentadas por los modelos tradicionales en el reconocimiento de las personas con 2e, es indispensable, entonces, diseñar e implementar modelos de identificación más comprensivos y auténticos que consideren las formas en que se expresan estas dualidades, y consideren múltiples dimensiones e informantes.

Desde el área de la intervención, emergen desde la evidencia empírica tres grandes necesidades: (a) comprender cómo los descensos en lo cognitivo ayudan a explicar las debilidades que pueden presentarse en lo académico, (b) entender que las altas habilidades presentadas en estudiantes $2 \mathrm{e}$ no siempre igualan las habilidades consideradas "exitosas" a nivel escolar y (c) crear programas efectivos de intervención basados en las fortalezas del estudiantado. Esto último es crucial, ya que habitualmente se brindan apoyos desde el déficit donde el estudiantado se sienten estigmatizado y frustrado, y las estrategias utilizadas son vistas como muy simples y poco desafiantes. Un enfoque basado en fortalezas puede incidir, no solo en mejorar el rendimiento, sino también la autoestima y la vinculación con el ámbito escolar.

\section{Agradecimientos}

Proyecto con financiamiento del Fondo Nacional de Desarrollo Científico y Tecnológico (Código Proyecto: FONDECYT 1151030, 2015-2017).

\section{REFERENCIAS}

Antshel, K. M., Faraone, S. V., Stallone, K., Nave, A., Kaufmann, F. A., Doyle, A., ... Biederman, J. (2007). Is attention deficit hyperactivity disorder a valid diagnosis in the presence of high IQ? Results from the MGH Longitudinal Family Studies of ADHD. Journal of Child Psychology and Psychiatry, 48(7), 687-694. doi: $10.1111 /$ j.1469-7610.2007.01735 
Assouline, S. G., Foley-Nicpon, M., Colangelo, N., y O’brien, M. (2008). The paradox of Giftedness and Autism: Packet of information for professionals. Iowa City: University of Iowa, College of Education.

Assouline, S. G., Foley-Nicpon., y Doobay, A. (2009). Profoundly gifted girls and autism spectrum disorder: A psychometric case study comparison. Gifted child quarterly, 53(2), 89-105. doi: 10.1177/0016986208330565

Assouline, S. G., Nicpon, M. F., y Dockery, L. (2012). Predicting the academic achievement of gifted students with autism spectrum disorder. Journal of autism and developmental disorders, 42(9), 1781-1789. doi: 10.1007/ s10803-011-1403-x

American Psychiatric Association. (2013). Diagnostic and statistical manual of mental disorders (5th ed.). Washington, DC: Author.

Barkley, R. A. (Ed.). (2015). Attention-deficit hyperactivity disorder: A handbook for diagnosis and treatment. New York: Guilford Publications.

Barnard-Brak, L., Johnsen, S. K., Pond Hannig, A., y Wei, T. (2015). The incidence of potentially gifted students within a special education population. Roeper Review, 37(2), 74-83. doi: 10.1080/02783193.2015.1008661

Baum, S. (2004). Introduction to Twice-Exceptional and Special Populations of Gifted Students. In S. Baum (Ed.), Twice-Exceptional and Special Populations of Gifted Students (Vol.7, pp. xxiii-xxxiii). California: Corwin Press.

Baum, S. M., Schader, R. M., y Hébert, T. P. (2014). Through a Different Lens Reflecting on a Strengths-Based, Talent-Focused Approach for Twice-Exceptional Learners. Gifted Child Quarterly, 58(4), 311-327. doi: $10.1177 / 0016986214547632$

Baum, S., Schader, R., y Owen, S. (2017). To Be Gifted \& Learning Disabled: Strength-Based Strategies for Helping Twice-exceptional Students with LD, ADHD, ASD and More (3th ed). Waco, Texas: Prufrock Press Inc.

Bélanger, J., y Gagné, F. (2006). Estimating the size of the gifted/talented population from multiple identification criteria. Journal for the Education of the Gifted, 30(2), 131-163. doi: 10.4219/jeg-2006-258

Bianco, M., y Leech, N. L. (2010). Twice-exceptional learners: Effects of teacher preparation and disability labels on gifted referrals. Teacher Education and Special Education: The Journal of the Teacher Education Division of the Council for Exceptional Children, 33(4), 319-334. doi: 10.1177/0888406409356392

Brown, T. E., Reichel, P. C., y Quinlan, D. M. (2011). Executive function impairments in high IQ children and adolescents with ADHD. Open Journal of Psychiatry, 1(2), 56-65.

Burger-Veltmeijer, A. E., Minnaert, A. E., y Van Den Bosch, E. J. (2015). Evaluación de alumnos de altas capacidades intelectuales con o sin características de TEA: Una exploración entre diagnosticadores de diversas organizaciones psicoeducativas. Electronic Journal of Research in Educational Psychology, 13(1), 5-26. doi: 10.14204/ ejrep.35.14109

Cash, A. B. (1999). A profile of gifted individuals with autism: The twice exceptional learner. Roeper Review, 22(1), 22-26.

Conejeros, M. L., Cáceres, P., y Riveros, A. (2012). Educación de talentos académicos en Chile: Una década de aprendizajes e investigación. En J. Catalán (Ed.), Investigación orientada al cambio en psicología educacional (pp.39-74). La Serena: Editorial Universidad de La Serena.

Conejeros-Solar, M. L., Gómez-Arizaga, M, P., Sandoval, K., Cáceres, P., Armijo, S., y Abello, I. (2016). Twice Exceptional students: Challenges in the Identification Process. Poster presented at the 15th International ECHA Conference. Talents in Motion, Viena, Austria.

Dabrowski, K. (1966). The theory of positive disintegration. International Journal of Psychiatry, 2(2), 229-249.

Daniels, S., y Piechowski, M. M. (2009). Living with intensity: Understanding the sensitivity, excitability, and emotional development of gifted children, adolescents, and adults. Tucson: Great Potential Press.

De La Barra, F., Vicente, B., Saldivia, S., y Melipillan, R. (2015). Epidemiología del TDAH en niños y adolescentes chilenos. Revista Chilena de Psiquiatría, 26(1), 34-46.

Donnelly, J. A., y Altman, R. (1994). The autistic savant: Recognizing and serving the gifted student with autism. Roeper Review, 16(4), 252-256. doi:10.1080/02783199409553591

Elkind, J. (1973). The gifted child with learning disabilities. Gifted Child Quarterly, 17, 96-97. 
Foley-Nicpon, M. (2015). Voices From the Field. The Higher Education Community. Gifted Child Today, 38(4), 249-251. doi: 10.1177/1076217515597288

Foley-Nicpon, M., Allmon, A., Sieck, B., y Stinson, R. (2011). Empirical Investigation of Twice- Exceptionality: Where Have We Been and Where Are We Going? Gifted Child Quarterly, 55(1), 3-17. doi: $10.1177 / 001698621038257$.

Foley-Nicpon, M., y Assouline, S. G. (2010a). Atendiendo a las necesidades de estudiantes talentosos con trastornos del espectro de autismo: Aproximaciones diagnósticas, terapéuticas y psicoeducativas. Psicoperspectivas, 9(2), 202-223. doi:10.5027/PSICOPERSPECTIVAS-VOL9-ISSUE2-FULLTEXT-87

Foley-Nicpon, M., Assouline, S. G., Amend, E. R., y Schuler, P. (2010b). Gifted and talented students on the autism spectrum: Best practices for fostering talent and accommodating concerns. En J. Castellano, y A. Frazier (Eds.), Special populations in gifted education: Understanding our most able students from diverse backgrounds (pp. 227-248). Waco: Prufrock Press.

Foley-Nicpon, M., Assouline, S. G., y Colangelo, N. (2013). Twice-exceptional learners: Who needs to know what? Gifted Child Quarterly, 57(3), 169-180. doi: 10.1177/0016986213490021

Foley-Nicpon, M., Assouline, S. G., y Stinson, R. D. (2012a). Cognitive and academic distinctions between gifted students with autism and Asperger syndrome. Gifted Child Quarterly, 56(2), 77-89. doi: $10.1177 / 0016986211433199$

Foley-Nicpon, M., Fosenburg, S. L., Wurster, K. G., y Assouline, S. G. (2017). Identifying high ability children with DSM-5 autism spectrum or social communication disorder: performance on autism diagnostic instruments. Journal of autism and developmental disorders, 47(2), 460-471. doi: 10.1007/s10803-016-2973-4

Foley-Nicpon, M., Rickels, H., Assouline, S. G., y Richards, A. (2012b). Self-Esteem and Self-Concept Examination Among Gifted Students with ADHD. Journal for the Education of the Gifted, 35(3), 220-240. doi: $10.1177 / 0162353212451735$

Fugate, C. M., Zentall, S. S., y Gentry, M. (2013). Creativity and working memory in gifted students with and without characteristics of attention deficit hyperactive disorder: Lifting the mask. Gifted Child Quarterly, 57(4), 234-246. doi: 10.1177/0016986213500069

Gómez, M, P., Conejeros-Solar, M. L., Sandoval, K., y Armijo, S. (2016). Doble excepcionalidad: Análisis exploratorio de experiencias y autoimagen en estudiantes chilenos. Revista de Psicologi\#a, 34(1), 5-37. doi: 10.18800/ psico.S01.001

Hartnett, D. N., Nelson, J. M., y Rinn, A. N. (2004). Gifted or ADHD? The possibilities of misdiagnosis. Roeper Review, 26(2), 73-76. doi: 10.1080/02783190409554245

Hébert, T. (2010). Understanding the Social and Emotional Lives of Gifted Students. Waco, Texas: Prufrock Press.

Heller, K. A. (2004). Identification of gifted and talented students. Psychology Science, 46(3), 302-323.

Hughes, C. E. (2011). Twice-exceptional children: Twice the challenges, twice the joys. Waco, TX: Prufrock Press.

Kalbfleisch, M. L. (2013). Twice-exceptional Students: Gifted Students with Learning Disabilities. In C. M. Callahan, y H. L. Hertberg-Davis (Ed.), Fundamentals of Gifted Education. Considering multiple perspectives (First ed., pp. 358-368). New York: Routledge.

Kalbfleisch, M. L., y Iguchi, C. M. (2008). Twice-exceptional learners. Critical issues and practices in gifted education. En J. A. Plucker, y C. M. Callahan (Eds.), Critical issues and practices in gifted education (pp.707-720). Waco, TX: Prufrock Press.

Krochak, L. A., y Ryan, T. G. (2007). The challenge of identifying gifted/learning disabled students. International Journal of Special Education, 22(3), 44-54.

Koshy, V., y Pascal, C. (2011). Nurturing the young shoots of talent: Using action research for exploration and theory building. European Early Childhood Education Research Journal, 19(4), 433-450. doi: $10.1080 / 1350293 X .2011 .623515$

Lee, K. M., y Olenchak, F. R. (2015). Individuals with a gifted/attention deficit/hyperactivity disorder diagnosis: Identification, performance, outcomes, and interventions. Gifted Education International, 31(3), 185-199. 
Little, C. (2002). Which is it? Aspergers syndrome or giftedness? Defining the differences. Gifted Child Today, 25(1), 58-64.

Lupart, J., y Toy, R. (2009). Twice Exceptional: Multiple Pathways to Success. In L. V. Shavinina (Ed.), International Handbook on Giftedness (pp. 507-525). Canada: Springer.

Manzano, A., Arranz, E., y Sánchez De Miguel, M. (2010). Multi-criteria identification of gifted children in a spanish sample. European Journal of Education and Psychology, 3(1), 5-17.

Marland, S. P. (1972). Education of the gifted and talented. Report to the Congress of the United States by the U. S. Commissioner of Education. Washington, DC: Government Printing Office.

McCallum, R. S., Bell, S. M., Coles, J. T., Miller, K. C., Hopkins, M. B., y Hilton-Prillhart, A. (2013). A model for screening twice-exceptional students (gifted with learning disabilities) within a response to intervention paradigm. Gifted Child Quarterly, 57(4), 209-222.

Melogno, S., Pinto, M. A., y Levi, G. (2015). Profile of the linguistic and metalinguistic abilities of a gifted child with autism spectrum disorder: A case study. Child Language Teaching and Therapy, 31(1), 113-126. doi: $10.1177 / 0265659014530414$

MINEDUC. (2009). Decreto supremo N.ำ170 Ley 20.201 Nueva subvención para niños y niñas con necesidades educativas especiales. Santiago: Autor.

Ministerio de Desarrollo Social. (2015). Segunda encuesta nacional de discapacidad. Recuperado de http://www.rostrosnuevos.cl/wp-content/uploads/2016/05/2016-05-04-Resultados-Poblaci\%C3\%B3ncon-discapacidad-mental-2do-ENDISC-Ministerio-Desarrollo-Social.pdf

Moon, S. M., Zentall, S. S., Grskovic, J. A., Hall, A., y Stormont, M. (2001). Emotional and social characteristics of boys with $\mathrm{AD} / \mathrm{HD}$ and giftedness: A comparative case study. Journal for the Education of the Gifted, 24(3), 207-247. doi: 10.1177/016235320102400302

Mullet, D. R., y Rinn, A. N. (2015). Giftedness and ADHD: Identification, Misdiagnosis, and Dual Diagnosis. Roeper Review, 37(4), 195-207. doi: 10.1080/02783193.2015.1077910

National Association for Gifted Children. (2009). Twice-Exceptionality. White Paper. Washington, DC. Recuperado de https://www.nagc.org/sites/default/files/Position\%20Statement/twice\%20exceptional.pdf

Neal, D., Matson, J. L., y Hattier, M. A. (2014). Validity of the autism spectrum disorder observation for children (ASD-OC). Journal of Mental Health Research in Intellectual Disabilities, 7, 14-33.

Neihart, M. (2000). Gifted Children with Asperger's Syndrome. Gifted Child Quarterly, 44(4), 222-230.

Newman, T. M., y Sternberg, R. J. (Eds.). (2004). Students with both gifts and learning disabilities. New York: Kluwer.

Peters, S. J., y Engerrand, K. G. (2016). Equity and Excellence. Proactive efforts in the identification of underrepresented students for gifted and talented services. Gifted Child Quarterly, 60(3), 159-171. doi: $10.1177 / 0016986216643165$

Pfeiffer, S.I. (2015a). Essentials of Gifted Assessment. New Jersey: Wiley.

Pfeiffer, S. (2015b). Gifted students with a coexisting disability: The twice-exceptional. Estudios de Psicología, 32(4), 717-727 doi: 10.1590/0103-166X2015000400015

Reis, S. M., Baum, S. M., y Burke, E. (2014). An Operational Definition of Twice-Exceptional Learners: Implications and Applications. Gifted Child Quarterly, 58(3), 217-230. doi: 10.1177/0016986214534976

Reis, S. M., y Ruban, L. M. (2004). Compensation strategies used by High-Ability with Learning Disabilities. In T. Newman y R. Sternberg (Ed.), Students with both Gifts and Learning Disabilities. Identification, Assessment, and Outcomes (Vol. 25, pp. 155-198). New York: Springer Science+Business Media.

Rinn, A. N., y Reynolds, M. J. (2012). Overexcitabilities and ADHD in the Gifted: An Examination. Roeper Review, 34(1), 38-45. doi: 10.1080/02783193.2012.627551

Ruban, L. M., y Reis, S. (2005). Identification and assessment of gifted students with learning disabilities. Theory into practice, $44(2), 115-124$. 
Rubenstein, L. D., Schelling, N., Wilczynski, S. M., y Hooks, E. N. (2015). Lived experiences of parents of gifted students with Autism Spectrum Disorder: The struggle to find appropriate educational experiences. Gifted Child Quarterly, 59(4), 283-298 doi: 10.1177/0016986215592193

Schultz, S. M. (2012). Twice-exceptional students enrolled in advanced placement classes. Gifted Child Quarterly, 56(3), 119-133. doi: 10.1177/0016986212444605

Silverman, L. (1998). Through the lens of giftedness. Roeper Review, 20, 204-210.

Sternberg, R., y Grigorenko, E. (2002). The Theory of Successful Intelligence as a Basis for Gifted Education. Gifted Child Quarterly, 46(4), 265-277.

Tapia Pérez, M. J., Veliz de Vos, M., y Reyes Reyes, F. (2017). Desempeño ejecutivo y rendimiento lector en estudiantes con trastorno por déficit de atención con hiperactividad. Logos, 27(1), 3-14. doi: 10.15443/rl2701

Tavani, J. L., Zenasni, F., y Pereira-Fradin, M. (2009). Social representation of gifted children: A Preliminary study in France. Gifted and Talented International, 24(2), 61-70.

Townend, G., Pendergast, D., y Garvis, S. (2014). Academic self-concept in twice-exceptional students: What the literature tells us. TalentEd, 28(1-2), 75-89.

Trail, B. (2011). Twice Exceptional Gifted Children. Understanding, Teaching, and Counseling Gifted Students. USA: Prufrock Press Inc.

UNESCO. (2002). Declaración de la Habana. Proyecto Regional de Educación para América Latina y el Caribe. Recuperado de http://www.systac.cl/archivospsp/habana.pdf

Whitaker, A. M., Bell, T. S., Houskamp, B. M., y O'Callaghan, E. T. (2015). A neurodevelopmental approach to understanding memory processes among intellectually gifted youth with attention-deficit hyperactivity disorder. Applied Neuropsychology: Child, 4(1), 31-40.

Withmore, J. R., y Maker, C. J. (1985). Intellectual giftedness in disabled persons. Austin, TX: Pro-Ed.

Ziemann, S. (2009). Merely bright-or brilliant? Finding out whether your child is gifted is the first step in getting her the challenge she craves. Recuperado de http://articles.chicagotribune.com/1999-09-19/ features/9909190194_1_parents-of-gifted-kids-giftedness-bright-child

Zentall, S. S., Moon, S. M., Hall, A. M., y Grskovic, J. A. (2001). Learning and motivational characteristics of boys with $\mathrm{AD} / \mathrm{HD}$ and/or giftedness. A multiple case study. Exceptional Children, 67, 499-519.

\section{BY-NC-ND}

